\title{
Macelignan inhibits bee pathogenic fungi Ascophaera apis growth through HOG1 pathway
}

\author{
Y.K. Shin ${ }^{1}$ and K.Y. Kim ${ }^{2}$ \\ ${ }^{1}$ College of Life Science, Kyung Hee University, Yongin-si, Gyeonggi-do, Korea \\ ${ }^{2}$ Department of Genetic Engineering, College of Life Science and Graduate School of Biotechnology, \\ Kyung Hee University, Yongin-si, Gyeonggi-do, Korea
}

\begin{abstract}
Ascosphaera apis is a bee pathogen that causes bee larvae infection disease, to which treatment is not yet well investigated. The aim of this study was to investigate antifungal susceptibility in vitro against $A$. apis and to identify a new antifungal agent for this pathogen through minimal inhibitory concentration (MIC) assay and western blot analysis. Macelignan had 1.56 and 3.125 $\mu \mathrm{g} / \mathrm{mL}$ MIC against $A$. apis after 24 and $48 \mathrm{~h}$, respectively, exhibiting the strongest growth inhibition against $A$. apis among the tested compounds (corosolic acid, dehydrocostus lactone, loganic acid, tracheloside, fangchinoline and emodin-8-O- $\beta$-Dglucopyranoside). Furthermore, macelignan showed a narrow-ranged spectrum against various fungal strains without any mammalian cell cytotoxicity. In spite of miconazole having powerful broad-ranged anti-fungal activity including $A$. apis, it demonstrated strong cytotoxicity. Therefore, even if macelignan alone was effective as an antifungal agent to treat $A$. apis, combined treatment with miconazole was more useful to overcome toxicity, drug resistance occurrence and cost effectiveness. Finally, HOG1 was revealed as a target molecule of macelignan in the anti- $A$. apis activity by inhibiting phosphorylation using $S$. cerevisiae as a model system. Based on our results, macelignan, a food-grade antimicrobial compound, would be an effective antifungal agent against $A$. apis infection in bees.
\end{abstract}

Key words: Bee; Ascosphaera apis; Macelignan; Antifungal agent; HOG1

\section{Introduction}

Pollination is a very important process for reproduction of many crops, fruit trees, and wild plants. Among pollinators, honeybees play a major role and are the most economically valuable pollination mediators for agricultural and horticultural plants worldwide. Due to their role in nature, honeybees have a great economic impact (1).

Honey bees are continually exposed to numerous threats: pests and parasites (such as the Varroa mite or Nosema), bacterial diseases (foulbrood), fungal diseases (chalkbrood), viral diseases (invertebrate iridescent virus), and pesticides. Even if the reduction of the bee population is not fully understood, microorganism infections are one of the main reasons. Chalkbrood is a bee larvae infection disease caused by the fungus Ascosphaera apis. Spores of this fungus grow within the digestive track of infected bees and then develop into the mycelial form. This causes the death of the larva, which will have the appearance of chalk. The chalky larva is highly infective to the other larvae within the nest $(2,3)$. A. apis can also infect adult bumble bees, which increases the potential to infect native bees via pathogen spillover (4). Except for the natural essential oils (5), no other compound that inhibits $A$. apis growth is reported.

Natural plants and medicinal herbs are a valuable resource for novel anti-microbiological agents because many have abundant biological activities and are pharmacologically safe (6-8). Natural compounds for the control of chalkbrood fungus would be a welcome alternative to synthetic fungicides. A broad range of compounds have been tested in honey bee colonies and on $A$. apis culture in an attempt to find a control for chalkbrood (2). Some of these compounds are natural plant-derived antimicrobial products $(2,5,9,10)$. Essential oils containing citral, geraniol and citronellal were reported to have the best inhibiting effect on fungal growth in vitro (5). However, most herb extracts showed their best inhibitory effects at relatively low concentrations. For example, 1.5-3.5\% (w/v) of Cinnamomum cassia and Piper betel extracts inhibited fungal growth (10) and more than $0.025 \%(\mathrm{w} / \mathrm{v})$ of essential oils were used for chalkbrood control (5).

Since single compounds showed better efficacy and controllable activity compared with crude extract, we are focusing on highly effective single compounds for

Correspondence: K.Y. Kim: <kiyoung@khu.ac.kr> 
chalkbrood disease treatment. In this paper, we tried to identify a putative antifungal compound against $A$. apis among the elements from natural plants. Our medicinal herb extracts pool was screened to find new functional antifungal agents.

\section{Material and Methods}

\section{Strains}

Aspergillus niger (KACC42589), Aspergillus clavatus (KACC40071), Candida parapsilosis var. parapsilosis (KACC45480), Rhizopus oryzae (KACC40256) and Saccharomyces cerevisiae (KACC30068) were obtained from KACC (Korea Agricultural Culture Collection). C. albicans (KCTC7965, KCTC7270), C. tropicalis (KCTC7212), C. tropocalis var. tropicalis (KCTC17762), C. glabrata (KCTC7219), Cryptococcus neoformans (KCTC17528), and Pichia guilliermondii (KCTC7211) were purchased from KCTC (Korea Collection for Type Cultures). Ascosphaera apis was a gift from Byungsoo Yun (Kyunggi University). S. cerevisiae (BY4742) was purchased from Life Technologies (USA).

A. apis was maintained with SDA medium (11-13) at $35^{\circ} \mathrm{C}$ containing ampicillin $(100 \mu \mathrm{g} / \mathrm{mL})$ under normal atmosphere. Growing fungi were regularly monitored for contamination. Other molds and yeasts were maintained in yeast peptone dextrose (YPD) plates.

\section{Broth microdilution antifungal testing}

For $A$. apis, the broth microdilution assay suggested by the European Committee on Antimicrobial Susceptibility Testing (EUCAST) was applied. Stock compound solutions were prepared in dimethyl sulfoxide (DMSO) with $10 \mathrm{mg} / \mathrm{mL}$ concentration. The compound was serially two-fold diluted with Sabouraud dextrose (SD; TaKaRa, Japan), Sabouraud dextrose $+0.2 \%$ yeast extract (SDYE), YPD (TaKaRa) or RPMI medium (RPMI 164, Sigma, USA) without sodium bicarbonate and with L-glutamine buffered to $\mathrm{pH} 7.0$ with $0.165 \mathrm{M}$ morpholinopropanesulfonic acid and added with $18 \mathrm{~g}$ of glucose per liter to make a final concentration of $2 \%(11-15)$. One hundred microliter of the medium containing each compound ranging from $400-0.39 \mu \mathrm{g} / \mathrm{mL}$ were added to the 96-well flat-bottomed microtitration plates (SPL, Korea). The last well was used for sterility and growth controls. One hundred microliter of the mold inoculum suspension containing $1 \times 10^{5}$ spores $/ \mathrm{mL}$ were added to each well of microdilution plates. Plates were incubated at $35^{\circ} \mathrm{C}$ for 24 and $48 \mathrm{~h}$. Minimum inhibitory concentration (MIC) values were defined as the lowest concentration of drug that completely inhibited cell growth.

For aspergillus species, the same broth microdilution assay described for $A$. apis antifungal testing was used, but with RPMI 1640 medium $(14,16)$.

For Candida and Saccharomyces species, the broth microdilution assay recommended in the NCCLS document
M27-A was used as described previously (15-18). Each compound (concentration ranged from 200-0.2 $\mu \mathrm{g} / \mathrm{mL}$ ) was serially two-fold diluted with $100 \mu \mathrm{L}$ of RPMI 1640 medium. The diluted drug was dispensed into 96 well round bottom microdilution plates (SPL) and $100 \mu \mathrm{L}$ of yeast inoculum was added with a concentration of $1 \times 10^{4}$ cells/ $\mathrm{mL}$ to each well of microdilution plates. Plates were incubated at $35^{\circ} \mathrm{C}$ for 24 and $48 \mathrm{~h}$. Tests were performed at least three times.

\section{Antifungal synergy testing}

Antifungal synergy between miconazole and macelignan against $A$. apis was tested using the broth microdilution assay and the checkerboard method (19). After miconazole and macelignan serial two-fold dilution, macelignan was dispensed into a 96-well microtitre plate with total $100 \mu \mathrm{L}$ of drug containing SD medium per well. A. apis was inoculated with $100 \mu \mathrm{L}$ of the suspension containing $1 \times 10^{5}$ spores $/ \mathrm{mL}$.

Final drug concentrations of $12.5-0.1 \mu \mathrm{g} / \mathrm{mL}$ for miconazole and of $12.5-0.05 \mu \mathrm{g} / \mathrm{mL}$ for macelignan were obtained. MIC values of individual drugs were determined on the same plate. Tests were performed at least three times.

The fractional inhibitory concentration index $(\mathrm{FICl})$ was used to determine synergistic effects of compounds using the equation: $\mathrm{FICl}=(\mathrm{Ac} / \mathrm{Aa})+(\mathrm{Bc} / \mathrm{Ba})$ where $\mathrm{Ac}$ and $\mathrm{Bc}$ are the MICs of drugs $A$ and $B$ in combination, and $A a$ and $B a$ are the MICs of drugs alone. $\mathrm{FICl}$ values $\leqslant 0.5$ indicate synergy, and values $0.5-4$ indicate no interaction (19).

\section{Cytotoxicity assays}

The cytotoxicity of the test compounds was done using HepG2 cells with slight modifications from the previously reported procedure using a cell-based MTT assay (HepG2 cells were kindly provided by Prof. T.H. Yi, Kyung Hee University) (20). Cells were seeded at $1 \times 10^{4}$ cells per well in 96-well plates. After $24 \mathrm{~h}$ of incubation, the cells were washed with PBS and cultured in Dulbecco's modified Eagle's medium (DMEM) containing various concentrations of macelignan $(0-3 \mathrm{mM})$. After $48 \mathrm{~h}$ of incubation, MTT solution (3-(4,5-dimethyl-thiazol-2-yl)-2,5diphenyltetrazolium Bromide, Sigma) in PBS was added to a final concentration of $0.5 \mathrm{mg} / \mathrm{mL}$, followed by incubation for $3 \mathrm{~h}$ at $37^{\circ} \mathrm{C}$. After, the supernatant medium was removed, and cells were suspended in $100 \mu \mathrm{L}$ of DMSO for $10 \mathrm{~min}$. Absorbance was measured at $520 \mathrm{~nm}$ using a micro-plate reader (VersaMax, Molecular Devices, USA). The cell proliferation rates were calculated from the optical density (OD) readings and reported as percentages of the vehicle control.

\section{Western blot analysis of MPK1 and HOG1 \\ in S. cerevisiae}

For HOG1 analysis, yeast cells (S. cerevisiae, BY4742; $10^{8}$ cells $/ \mathrm{mL}$ ) were incubated with macelignan 
and $0.4 \mathrm{M} \mathrm{NaCl}$ for $1 \mathrm{~h}$. After disruption of the cells, $100 \mu \mathrm{g}$ total protein was extracted and separated with SDSPAGE. p-HOG1 and total HOG1 were detected with p-p38 (Santa Cruz, USA; sc-17852) and Hog1 antibody (Santa Cruz; sc-6815), respectively. For MPK1 analysis, cells with $10 \mu \mathrm{g} / \mathrm{mL}$ of macelignan were heated to $39^{\circ} \mathrm{C}$ to give heat stress. After $2 \mathrm{~h}$, cells were disrupted and $100 \mu \mathrm{g}$ of total protein were loaded to SDS-PAGE. p-MPK1 was detected with p42/44 MAPK (ERK1/2) antibody (Sigma) and MPK1-FLAG was detected with anti-FLAG antibody (M2; Sigma).

\section{Results}

\section{Natural plant extracts}

This study searched for a putative antifungal compound against $A$. apis among the elements from natural plants. First a pool of medicinal herb extracts was screened to find newly functional antifungal agents. The following nine plant extracts were identified: Crataegus pinnatifida (Bunge leaf), Rhododendron brachycarpum (fruit), the root of Polygonum multiflorum Thunb. (root), Cassia brewsteri (root), the root of Stephania tetrandra (root), Gentiana scabra Bunge (root), carthamus tinctorius Linne (seed), Saussurea lappa (root), and Schisandra chinensis (fruit). These extracts showed preventive effects for fungal cell growth but their effects were not strong enough (Data not shown).

\section{Single compound analysis for anti-A. apis activity}

Because of the negative results from the tested herb extracts, widely known active compounds of nine herbal extracts were purchased from Chemface (China) to find an effective antifungal agent. We selected macelignan, corosolic acid, dehydrocostus lactone, loganic acid, tracheloside, fangchinoline and emodin-8-O- $\beta$-D-glucopyranoside because of their unknown antifungal function. We applied SD, SDYE, YPD or RPMI1640 medium to test antifungal activity since the standard medium for broth microdilution assay of $A$. apis was not established yet. Luckily medium effects were not detected for MIC, thereby SD medium was used for anti-fungal assay (Data not shown). Finally, macelignan showed the strongest anti$A$. apis activity among all extracts (Table 1). Macelignan had 1.56 and $3.125 \mu \mathrm{g} / \mathrm{mL}$ MIC against $A$. apis after 24

Table 1. In vitro antifungal activity of various compounds against A. apis at 24 and $48 \mathrm{~h}$.

\begin{tabular}{lcc}
\hline \multirow{2}{*}{ Compounds } & \multicolumn{2}{c}{ MIC for A. apis } \\
\cline { 2 - 3 } & $24 \mathrm{~h}$ & $48 \mathrm{~h}$ \\
\hline Macelignan & 1.56 & 3.125 \\
Corosolic acid & 12.5 & 12.5 \\
Dehydrocostus lactone & 50 & 50 \\
Fangchinoline & 200 & $>200$ \\
Tracheloside & 100 & $>200$ \\
Loganic acid & 100 & $>200$ \\
Emodin-8-O- $\beta$-D-glucopyranoside & $>200$ & $>200$ \\
Miconazole & 1.56 & 1.56 \\
\hline
\end{tabular}

Data are reported as minimum inhibitory concentration (MIC) in $\mu \mathrm{g} / \mathrm{mL}$. The experiment was repeated three times with essentially the same results.

Table 2. In vitro susceptibility of fungi to macelignan at 24 and $48 \mathrm{~h}$.

\begin{tabular}{lccc}
\hline Strain code & Strain name & \multicolumn{2}{c}{ MIC for macelignan } \\
\cline { 2 - 4 } & & $24 \mathrm{~h}$ & $48 \mathrm{~h}$ \\
\hline KH A5 & A. apis & 1.56 & 3.125 \\
KACC42589 & A. niger & $>200$ & $>200$ \\
KACC40071 & A. clavatus & $>200$ & $>200$ \\
KCTC7965 & C. albicans & $>200$ & $>200$ \\
KCTC7270 & C. albicans & $>200$ & $>200$ \\
KACC45480 & C. parapsilosis var. parapsilosis & $>200$ & $>200$ \\
KCTC7212 & C. tropicalis & $>200$ & $>200$ \\
KCTC17762 & C. tropocalis var. tropicalis & $>200$ & $>200$ \\
KCTC7219 & C. glabrata & $>200$ & $>200$ \\
KCTC17528 & C. neoformans & 6.25 & 6.25 \\
KCTC7211 & P. guilliermondii & 3.125 & 200 \\
KACC40256 & R. oryzae & $>200$ & $>200$ \\
BY4742 & S. cerevisiae & $>200$ & $>200$ \\
KACC30068 & S. cerevisiae & $>200$ & $>200$ \\
\hline
\end{tabular}

Data are reported as minimum inhibitory concentration (MIC) in $\mu \mathrm{g} / \mathrm{mL}$. The experiment was repeated three times with essentially the same results. 
and $48 \mathrm{~h}$, respectively. Corosolic acid $(12.5 \mu \mathrm{g} / \mathrm{mL}$ MIC after 24 and $48 \mathrm{~h})$ and dehydrocostus lactone $(50 \mu \mathrm{g} / \mathrm{mL}$ MIC after 24 and $48 \mathrm{~h}$ ) had anti- $A$. apis activity but their effects were weaker than macelignan. In addition, miconazole was used as a positive control, and showed very high antifungal effects against $A$. apis $(1.56 \mu \mathrm{g} / \mathrm{mL}$ MIC after 24 and $48 \mathrm{~h}$ ).

\section{Broad spectrum analysis}

We explored whether macelignan, corosolic acid, dehydrocostus lactone and miconazole had broad spectrum against various fungus including $A$. niger, A. clavatus, C. parapsilosis var. parapsilosis, R. oryzae, S. cerevisiae, C. albicans, C. tropicalis, C. tropocalis var. tropicalis, C. glabrata, C. neoformans and $P$. guilliermondii. Interestingly, macelignan showed a very narrow spectrum against various tested fungus. Macelignan had anti-fungal activity specifically to $A$. apis, $C$. neoformans and $P$. guilliermondii (MIC $=3.125,6.25$ and $3.125 \mu \mathrm{g} / \mathrm{mL}$ after $24 \mathrm{~h}$, respectively; Table 2). After $48 \mathrm{~h}$, macelignan still showed $3.125 \mu \mathrm{g} / \mathrm{mL}$ MIC against $A$. apis and $6.25 \mu \mathrm{g} / \mathrm{mL}$ MIC against $C$. neoformans, but 200 $\mu \mathrm{g} / \mathrm{mL}$ MIC against $P$. guilliermondii (Table 2).

Corosolic acid showed a mild effect against $A$. apis $(12.5 \mu \mathrm{g} / \mathrm{mL}$ MIC after 24 and $48 \mathrm{~h}$, Table 3). Even if corosolic acid showed strong anti-C. neoformans activity (3.125 $\mu \mathrm{g} / \mathrm{mL}$ MIC) after $24 \mathrm{~h}$, MIC after $48 \mathrm{~h}$ was decreased to over $200 \mu \mathrm{g} / \mathrm{mL}$. Unfortunately, dehydrocostus lactone did not show strong effects, but a weak antifungal activity against all of the tested fungus was observed (Table 4). MIC values for miconazole ranged from 1.56 to $25 \mu \mathrm{g} / \mathrm{mL}$ against all of tested fungus after 24- to 48-h incubation (Table 5).

\section{Synergistic inhibition of $\boldsymbol{A}$. apis by macelignan and miconazole}

We applied the broth microdilution assay in a checkerboard method to determine if miconazole and macelignan synergistically inhibited the growth of $A$. apis (Table 6). Despite miconazole and macelignan not showing strong synergistic effects against $A$. apis $(\mathrm{FICl}=0.75$ and 0.78 after 24- and 48-h incubation, respectively), miconazole MIC was reduced to $0.78 \mu \mathrm{g} / \mathrm{mL}$ combined with $0.39 \mu \mathrm{g} / \mathrm{mL}$ of macelignan. We also observed a weak growth of A. apis at $0.78 \mu \mathrm{g} / \mathrm{mL}$ miconazole with all macelignan concentrations tested and $0.78 \mu \mathrm{g} / \mathrm{mL}$ of macelignan with all miconazole concentrations tested.

\section{Cytotoxicity of macelignan and miconazole}

Cytotoxicity of macelignan and miconazole against HepG2 cells was tested to dertermine whether compounds also inhibit the growth of human cells (Figure 1). Macelignan had low cytotoxicity against HepG2 cells $\left(\mathrm{IC}_{50}=62.7 \pm 5.3 \mu \mathrm{g} / \mathrm{mL}\right.$; Figure $\left.1 \mathrm{~A}\right)$. This result is in agreement with the report that showed that macelignan had weak cytotoxicity against the HepG2 and MDA cells (21). However, miconazole had a strong cytotoxicity $\left(\mathrm{IC}_{50}=5.9 \pm 5.5 \mu \mathrm{g} / \mathrm{mL}\right.$; Figure 1B).

\section{Anti-A. apis target validation using $S$. cerevisiae}

Although $A$. apis genome sequencing was completed at 2006, translational analysis was barely performed because of poor antibody availability against $A$. apis. Therefore, in this study $S$. cerevisiae was used to validate $A$. apis antifungal target. Initially, we used the $A$. apis genome sequencing project results. We found two candidate genes Scaffold65 and Scaffold592, both of

Table 3. In vitro susceptibility of fungi to corosolic acid at 24 and $48 \mathrm{~h}$.

\begin{tabular}{lccc}
\hline Strain code & Strain name & \multicolumn{2}{c}{ MIC for corosolic acid } \\
\cline { 2 - 4 } & & $24 \mathrm{~h}$ & $48 \mathrm{~h}$ \\
\hline KH A5 & A. apis & 12.5 & 12.5 \\
KACC42589 & A. niger & $>200$ & $>200$ \\
KACC40071 & A. clavatus & $>200$ & $>200$ \\
KCTC7965 & C. albicans & $>200$ & $>200$ \\
KCTC7270 & C. albicans & $>200$ & $>200$ \\
KACC45480 & C. parapsilosis var. parapsilosis & $>200$ & $>200$ \\
KCTC7212 & C. tropicalis & $>200$ & $>200$ \\
KCTC17762 & C. tropocalis var. tropicalis & $>200$ & $>200$ \\
KCTC7219 & C. glabrata & $>200$ & $>200$ \\
KCTC17528 & C. neoformans & 3.125 & $>200$ \\
KCTC7211 & P. guilliermondii & $>200$ & $>200$ \\
KACC40256 & R. oryzae & $>200$ & $>200$ \\
BY4742 & S. cerevisiae & $>200$ & $>200$ \\
KACC30068 & S. cerevisiae & $>200$ & $>200$ \\
\hline
\end{tabular}

Data are reported as minimum inhibitory concentration (MIC) in $\mu \mathrm{g} / \mathrm{mL}$. The experiment was repeated three times with essentially the same results. 
Table 4. In vitro susceptibility of fungi to dehydrocostus lactone at 24 and $48 \mathrm{~h}$.

\begin{tabular}{lcrr}
\hline Strain code & Strain name & \multicolumn{2}{c}{ MIC for dehydrocostus lactone } \\
\cline { 2 - 4 } & & $24 \mathrm{~h}$ & $48 \mathrm{~h}$ \\
\hline KH A5 & A. apis & 50 & 50 \\
KACC42589 & A. niger & 100 & 100 \\
KACC40071 & A. clavatus & 50 & 50 \\
KCTC7965 & C. albicans & 100 & $>200$ \\
KCTC7270 & C. albicans & 200 & 200 \\
KACC45480 & C. parapsilosis var. parapsilosis & 100 & 200 \\
KCTC7212 & C. tropicalis & 50 & 50 \\
KCTC17762 & C. tropocalis var. tropicalis & 50 & $>200$ \\
KCTC7219 & C. glabrata & 100 & 100 \\
KCTC17528 & C. neoformans & 25 & 100 \\
KCTC7211 & P. guilliermondii & 50 & 50 \\
KACC40256 & R. oryzae & $>200$ & $>200$ \\
BY4742 & S. cerevisiae & 100 & 100 \\
KACC30068 & S. cerevisiae & 50 & 50 \\
\hline
\end{tabular}

Data are reported as minimum inhibitory concentration (MIC) in $\mu \mathrm{g} / \mathrm{mL}$. The experiment was repeated three times with essentially the same results.

Table 5. In vitro susceptibility of fungi to miconazole at 24 and $48 \mathrm{~h}$.

\begin{tabular}{lccc}
\hline Strain code & Strain name & \multicolumn{2}{c}{ MIC for miconazole } \\
\cline { 2 - 4 } & & $24 \mathrm{~h}$ & $48 \mathrm{~h}$ \\
\hline KH A5 & A. apis & 3.125 & 3.125 \\
KACC42589 & A. niger & 6.25 & 12.5 \\
KACC40071 & A. clavatus & 12.5 & 12.5 \\
KCTC7965 & C. albicans & 1.56 & 12.5 \\
KCTC7270 & C. albicans & 1.56 & 12.5 \\
KACC45480 & C. parapsilosis var. parapsilosis & 12.5 & 12.5 \\
KCTC7212 & C. tropicalis & 12.5 & 12.5 \\
KCTC17762 & C. tropocalis var. tropicalis & 25.0 & 25.0 \\
KCTC7219 & C. glabrata & 12.5 & 12.5 \\
KCTC17528 & C. neoformans & 6.25 & 3.125 \\
KCTC7211 & P. guilliermondii & 12.5 & 12.5 \\
KACC40256 & R. oryzae & $\mathrm{nt}$ & $\mathrm{nt}$ \\
BY4742 & S. cerevisiae & 12.5 & 25 \\
KACC30068 & S. cerevisiae & 12.5 & 12.5 \\
\hline
\end{tabular}

Data are reported as minimum inhibitory concentration values (MIC) in $\mu \mathrm{g} / \mathrm{mL}$. The experiment was repeated three times with essentially the same results. nt: not tested.

which have short sequences. Compared with $S$. cerevisiae HOG1 that has 435 amino acids, predicted amino acid numbers for Scaffold65 is 228 and for the Scaffold592 is 94 . But when we examined their sequences, we found that both are actually the same, and differences were because of the mRNA quality; moreover, there were
2 un-sequenced regions. Therefore, we prepared the genomic DNA of the $A$. apis to inspect its sequence, and generated the predicted amino acid sequence for $A$. apis HOG1 homologue. We performed an alignment with 3 different programs that showed $79.5 \%$ identity with $89.7 \%$ similarity at the conserved kinase region and 
Table 6. Synergistic antifungal effects of macelignan combined with miconazole against $A$. apis indicated by minimum inhibitory concentration (MIC) and fractional inhibitory concentration index ( $\mathrm{FICl})$.

\begin{tabular}{|c|c|c|c|c|c|c|}
\hline & \multicolumn{4}{|c|}{ MIC $(\mu \mathrm{g} / \mathrm{mL})$} & \multicolumn{2}{|c|}{$\mathrm{FICl}$} \\
\hline & \multicolumn{2}{|c|}{ Macelignan } & \multicolumn{2}{|c|}{ Miconazole } & \multirow[b]{2}{*}{$24 \mathrm{~h}$} & \multirow[b]{2}{*}{$48 \mathrm{~h}$} \\
\hline & $24 \mathrm{~h}$ & $48 \mathrm{~h}$ & $24 \mathrm{~h}$ & $48 \mathrm{~h}$ & & \\
\hline Representative value & 1.56 & 3.125 & 1.56 & 1.56 & $0.75 \pm 0.15$ & $0.78 \pm 0.14$ \\
\hline
\end{tabular}

MIC values are reported as representative value in $\mu \mathrm{g} / \mathrm{mL}$ and $\mathrm{FICl}$ values are reported as means \pm SD. The experiment was repeated three times with essentially the same results.
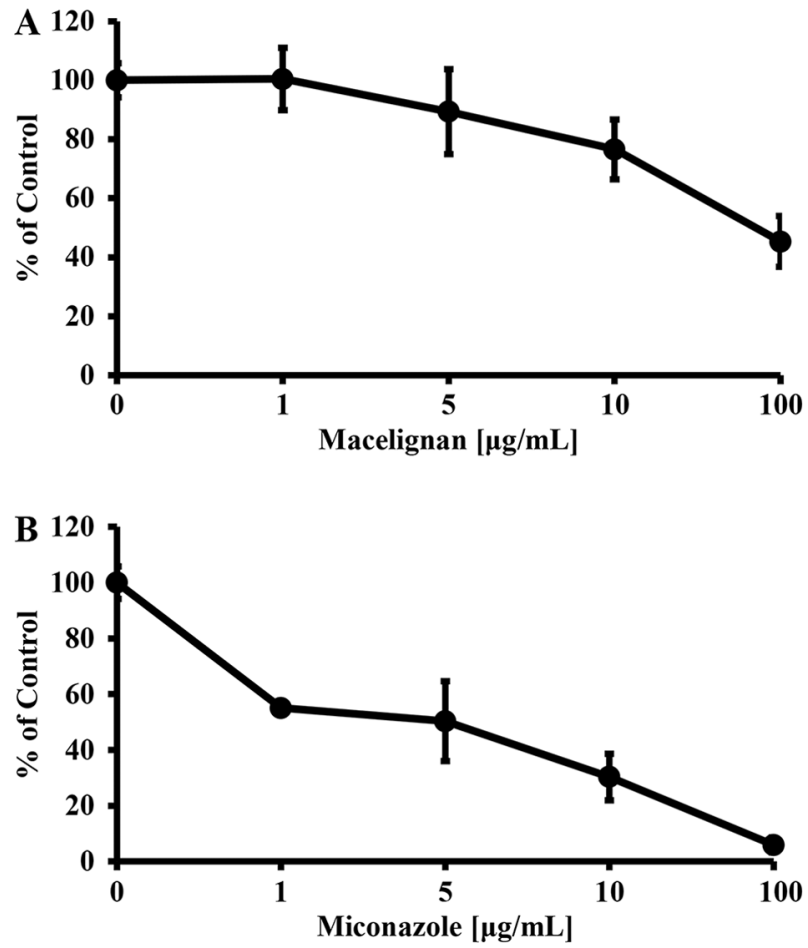

Figure 1. Cytotoxic effects of the macelignan $(A)$ and miconazole $(B)$ against hepG2 cells. HepG2 cells were treated with the indicated concentrations of macelignan and miconazole for $24 \mathrm{~h}$. Data are reported as means \pm SD of three independent experiments.

$63.7 \%$ identity if the c-terminal variable region was included. Therefore, we conclude that putative $A$. apis HOG1 was very similar to $S$. cerevisiae HOG1. Total protein and phosphorylated forms of MPK and HOG1 were observed after macelignan treatment (Figure 2). After macelignan treatment, phosphorylation of HOG1 showed a dose-dependent inhibition compared with total HOG1 protein. However, MPK1 phosphorylation was not

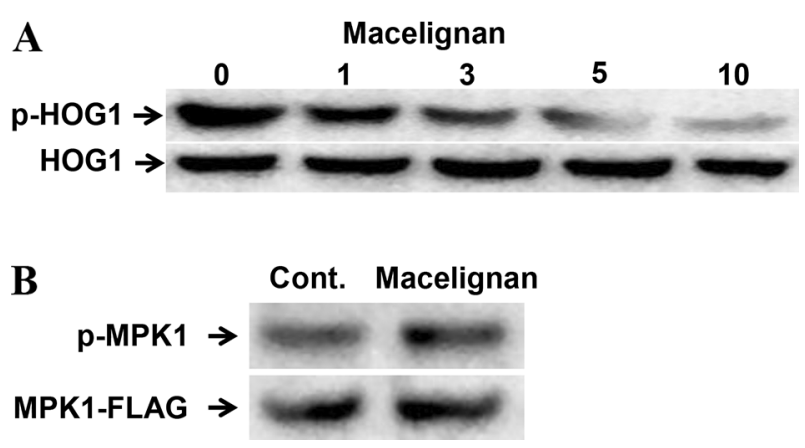

Figure 2. Western blot analysis of HOG1 and MPK1. $A$, S. cerevisiae was incubated with $1,3,5$ and $10 \mu \mathrm{g} / \mathrm{mL}$ macelignan for $1 \mathrm{~h}$ with $0.4 \mathrm{M} \mathrm{NaCl}$. Proteins were then extracted and detected for phosphorylated and total HOG1. Total HOG1 protein was used as a loading control (Cont.). B, S. cerevisiae with $10 \mu \mathrm{g} / \mathrm{mL}$ macelignan was incubated at $39^{\circ} \mathrm{C}$ for $2 \mathrm{~h}$ to activate MPK1. Proteins were then extracted and detected for phosphorylated and total MPK1. Total MPK1 protein was used as a loading control. Images are representative of at least three independent experiments.

changed even with the highest concentration of macelignan treatment.

\section{Discussion}

In the present study, macelignan exhibited the strongest growth inhibition against $A$. apis among tested compounds, followed by corosolic acid and dehydocostus lactone. Miconazole also had anti- $A$. apis activity. In addition, miconazole MIC value was reduced when combined with macelignan. Although macelignan was effective as a sole antifungal agent to treat $A$. apis, combined treatment with miconazole showed much better results.

Miconazole is an imidazole antifungal agent, which is commonly applied topically on the skin or to mucous membranes for treating fungal infections in human and veterinary medicine. It works by inhibiting the synthesis of ergosterol, a critical component of fungal cell membranes. 
Even though miconazole is a well-known antifungal drug and is relatively cheap, miconazole has never been reported for $A$. apis growth inhibition. In spite of miconazole having powerful anti-fungal activity, it has strong cytotoxicity (22-24). Therefore, combination of miconazole and macelignan would strengthen the cost-effectiveness of miconazole while overcoming the cytotoxicity by the usage of a natural origin compound - macelignan.

Previously developed antimicrobial compounds are usually active against various microorganisms. Overuse of broad-spectrum anti-microbial compounds facilitates the development of antibiotic-resistant infections and multidrug resistance. Moreover, growth of probiotic organisms, which help make the microbial flora healthier, is also inhibited by anti-microbial drugs. In other words, when broad range anti-microbial agents are applied to control the pathogenic microorganism, these agents also suppress the growth of favorable organisms. Accordingly, one of the trends in the development of anti-microbial agents is to find drugs that are narrow-ranged (25). Therefore, the narrow spectrum of macelignan would be advantageous to treat a fungal disease in honey bees.

Macelignan is a natural phenolic compound derived from Myristica fragrans (nutmeg) (26). It possesses many biological properties, including antioxidant, anti-inflammatory, antibacterial, hepato-protective and neuro-protective activities (27-31). The molecular mechanisms for some of these biological effects have been explored in recent years, providing a foundation for understanding the pharmacological actions of macelignan (32). For example, macelignan suppressed the mitogen activated protein kinase (MAPK) signaling pathway, including p38, for the anti-inflammatory effect (33). Macelignan also protected cisplatin-induced hepato-toxicity through JNK1/2 and ERK1/2 de-phosphorylation (31) and regulated ROS-induced MAPK signaling in human skin fibroblasts (34).

MAPK cascade transmits signals from outer cell surface to the nucleus and is involved in fungal survival mechanisms against environmental stress conditions (35). MAPK signaling molecules can be good targets for antifungal drugs to avoid fungal survival against conventional drugs. Heitman's lab published research reporting that MPK1 and HOG1, MAPK pathway molecules, have antifungal drug

\section{References}

1. Bradbear N. Non-wood forest products 19, Bees and their role in forest livelihoods; a guide to the services provided by bees and the sustainable harvesting, processing and marketing of their products Rome: Food and Agricultural Organization of the United Nations Rome; 2009.

2. Aronstein KA, Murray KD. Chalkbrood disease in honey bees. J Invertebr Pathol 2010; 103 (Suppl 1): S20-S29, doi: 10.1016/j.jip.2009.06.018. sensitivity in C. neoformans $(36,37)$. Jung and Bahn also examined stress-activated signaling pathways, including HOG and MPK1 pathways, that would be drug target components for treatment of $C$. neoformans (38). In the present study, macelignan had antifungal activity only against $C$. neoformans, other than $A$. apis, which might infer the inhibition of MPK1 and/or HOG pathways. Recently, a systems biology study confirmed MPK1 among 11 MAPK molecules as the best target for antifungal drugs (39). Therefore, inhibition of $A$. apis and C. neoformans growth by macelignan treatment can be assumed through regulation of MPK1 and/or HOG1 pathways. To validate which pathway was related with the anti-A. apis effect, $S$. cerevisiae was used for protein analysis because $A$. apis antibodies are not commercially available. When macelignan was used in MPK1 or HOG1 protein activated yeast cells, only HOG1 phosphorylation was inhibited dosedependently. Therefore, HOG1, homolog of mammalian p38, should be a target of macelignan.

In conclusion, macelignan exhibited a strong growth inhibition against $A$. apis, with a narrow range spectrum. When macelignan was combined with miconazole, each compensated the other's weakness, showing a synergistic effect. Macelignan, as a food-grade antimicrobial compound, would minimally influence the eco-system (40) and could be a good candidate for the treatment of bee diseases caused by microorganisms. However, macelignan is not inexpensive enough to be solely used compared with miconazole. Therefore, combined usage of macelignan and miconazole would compensate the relatively high cost of macelignan, the occurrence of antifungal-agent resistance and the cytotoxicity of miconazole. Field tests to confirm the effectiveness of macelignan will be performed, as well as the development of macelignan derivatives to increase compound stability and anti-A. apis activity.

\section{Acknowledgments}

We thank Byungsoo Yun (Kyunggi University) for kindly providing $A$. apis. This work was supported by a grant from the Kyung Hee University in 2013 (KHU20131805).
3. Garrido-Bailon E, Higes M, Martinez-Salvador A, Antunez K, Botias C, Meana A, et al. The prevalence of the honeybee brood pathogens Ascosphaera apis, Paenibacillus larvae and Melissococcus plutonius in Spanish apiaries determined with a new multiplex PCR assay. Microb Biotechnol 2013; 6: 731-739, doi: 10.1111/1751-7915.12070.

4. Maxfield-Taylor SA, Mujic AB, Rao S. First detection of the larval chalkbrood disease pathogen Ascosphaera apis 
(Ascomycota: Eurotiomycetes: Ascosphaerales) in adult bumble bees. PLoS One 2015; 10: e0124868, doi: 10.1371/journal.pone.0124868.

5. Davis C, Ward W. Control of chalkbrood disease with natural products: a report for the RIRDC. Kingston: Publication No. 03/107, ACT, AU; 2003.

6. Floris I, Carta C. In vivo activity of Cinnamomum zeylanicum Nees essential oil against Bacillus larvae White. Apicoltura 1990; 6: 57-61.

7. Albo GN, Henning C, Ringuelet J, Reynaldi FJ, De Giusti MR, Alippi AM. Evaluation of some essential oils for the control and prevention of American Foulbrood disease in honey bees. Apidologie 2003; 34: 417-427, doi: 10.1051/ apido:2003040.

8. Gende LB, Fernandez N, Buffa F, Ruiu L, Satta A, Fritz R, et al. Susceptibility of Paenibacillus larvae isolates to a tetracycline hydrochloride and Cinnamon (Cinnamomum zeylanicum) essential oil mixture. Bulletin of Insectology 2010; 63: 247250.

9. Mourad AK, Zaghloul OA, El Kady MB, Nemat FM, Morsy ME. A novel approach for the management of the chalkbrood disease infesting honeybee Apis mellifera $\mathrm{L}$. (Hymenoptera: Apidae) colonies in Egypt. Commun Agric Appl Biol Sci 2005; 70: 601-611.

10. Chantawannakul P, Puchanichanthranon T, Wongsiri S. Inhibitory effects of some medicinal plant extracts on the growth ofascosphaera apis. Acta Horticulturae 2005; 678: 183-189, doi: 10.17660/ActaHortic.2005.678.26.

11. Atlas RM. Handbook of microbiological media. Parks LC, Boca Raton: CRC Press; 1993.

12. Reinaldi FJ, De Giusti MR, Alippi AM. Inhibition of the growth of Ascosphaera apis by Bacillus and Paenibacillus strains isolated from honey. Rev Argentina Microbio 2004; 36: 52-55.

13. Subsomboon $\mathrm{T}$, Liewseree $\mathrm{P}$, Kositchaimongkol $\mathrm{S}$. Culture media for growth of mycelium and for induction of sporulation of Ascosphaera apis, the causative agent of chalkbrood disease of honey bee (Apis mellifera L.). J Sci Technol MSU 2012; 32: 51-55.

14. Lass-Florl C, Cuenca-Estrella M, Denning D, RodriguezTudela J. Antifungal susceptibility testing in Aspergillus spp. according to EUCAST methodology. Med Mycol 2006; 44: 319-325, doi: 10.1080/13693780600779401.

15. Kim KY, Shin YK, Park JC, Kim JH, Yang H, Han DM, et al. Molecular cloning and biochemical characterization of Candida albicans acyl-CoA:sterol acyltransferase, a potential target of antifungal agents. Biochem Biophys Res Commun 2004; 319: 911-919, doi: 10.1016/j.bbrc.2004. 05.076 .

16. Cuenca-Estrella $M$, Lee-Yang $W$, Ciblak MA, rthingtonSkaggs BA, Mellado E, Warnock DW, et al. Comparative evaluation of NCCLS M27-A and EUCAST broth microdilution procedures for antifungal susceptibility testing of candida species. Antimicrob Agents Chemother 2002; 46: 3644-3647, doi: 10.1128/AAC.46.11.3644-3647.2002.

17. National Committee for Clinical Laboratory Standards. Methods for dilution antimicrobial susceptibility tests for bacteria that grow aerobically. 5th edn. Approved standard. M7-A45. Wayne: NCCLS; 2000.

18. Park KS, Kang KC, Kim KY, Jeong PY, Kim JH, Adams DJ, et al. HWY-289, a novel semi-synthetic protoberberine derivative with multiple target sites in Candida albicans. J Antimicrob Chemother 2001; 47: 513-519, doi: 10.1093/ jac/47.5.513.

19. Drogari-Apiranthitou M, Mantopoulou FD, Skiada A, Kanioura L, Grammatikou M, Vrioni G, et al. In vitro antifungal susceptibility of filamentous fungi causing rare infections: synergy testing of amphotericin B, posaconazole and anidulafungin in pairs. $J$ Antimicrob Chemother 2012; 67: 1937-1940, doi: 10.1093/jac/dks137.

20. Shin HS, Park SY, Hwang ES, Lee DG, Mavlonov GT, Yi TH. Ginsenoside F2 reduces hair loss by controlling apoptosis through the sterol regulatory element-binding protein cleavage activating protein and transforming growth factor-beta pathways in a dihydrotestosterone-induced mouse model. Biol Pharm Bull 2014; 37: 755-763, doi: 10.1248/bpb. b13-00771.

21. Xu LJ, Huang F, Chen SB, Li LN, Chen SL, Xiao PG. A cytotoxic neolignan from Schisandra propinqua (Wall.) Baill. J Integrative Plant Biol 2006; 48: 1493-1497, doi: 10.1111/j.1744-7909.2006.00323.x.

22. Berry M, Gurung A, Easty DL. Toxicity of antibiotics and antifungals on cultured human corneal cells: effect of mixing, exposure and concentration. Eye 1995; 9 (Part 1): 110-115, doi: 10.1038/eye.1995.17.

23. Benko I, Hernadi F, Megyeri A, Kiss A, Somogyi G, Tegyey $Z$, et al. Comparison of the toxicity of fluconazole and other azole antifungal drugs to murine and human granulocytemacrophage progenitor cells in vitro. $J$ Antimicrob Chemother 1999; 43: 675-681, doi: 10.1093/jac/43.5.675.

24. Tondervik A, Sletta H, Klinkenberg G, Emanuel C, Powell LC, Pritchard MF, et al. Alginate oligosaccharides inhibit fungal cell growth and potentiate the activity of antifungals against Candida and Aspergillus spp. PLoS One 2014; 9: e112518, doi: 10.1371/journal.pone.0112518.

25. Bax R, Green S. Antibiotics: the changing regulatory and pharmaceutical industry paradigm. J Antimicrob Chemother 2015; 70: 1281-1284, doi: 10.1093/jac/dku572.

26. Woo WS, Shin K, Wagner $\mathrm{H}$, Lotter $\mathrm{H}$. The structure of macelignan fromMyristica fragrans. Phytochemistry 1987; 26: 1542-1543, doi: 10.1016/S0031-9422(00)81858-0.

27. Sadhu SK, Okuyama E, Fujimoto H, Ishibashi M. Separation of Leucas aspera, a medicinal plant of Bangladesh, guided by prostaglandin inhibitory and antioxidant activities. Chem Pharm Bull 2003; 51: 595-598, doi: 10.1248/cpb. 51.595.

28. Jin DQ, Lim CS, Hwang JK, Ha I, Han JS. Anti-oxidant and anti-inflammatory activities of macelignan in murine hippocampal cell line and primary culture of rat microglial cells. Biochem Biophys Res Commun 2005; 331: 1264-1269, doi: 10.1016/j.bbrc.2005.04.036.

29. Chung JY, Choo JH, Lee MH, Hwang JK. Anticariogenic activity of macelignan isolated from Myristica fragrans (nutmeg) against Streptococcus mutans. Phytomedicine 2006; 13: 261-266, doi: 10.1016/j.phymed.2004.04.007.

30. Cui CA, Jin DQ, Hwang YK, Lee IS, Hwang JK, Ha I, et al. Macelignan attenuates LPS-induced inflammation and reduces LPS-induced spatial learning impairments in rats. Neurosci Lett 2008; 448: 110-114, doi: 10.1016/ j.neulet.2008.10.035.

31. Sohn JH, Han KL, Kim JH, Rukayadi Y, Hwang JK. Protective effects of macelignan on cisplatin-induced 
hepatotoxicity is associated with JNK activation. Biol Pharm Bull 2008; 31: 273-277, doi: 10.1248/bpb.31.273.

32. Paul S, Hwang JK, Kim HY, Jeon WK, Chung C, Han JS. Multiple biological properties of macelignan and its pharmacological implications. Arch Pharm Res 2013; 36: 264-272, doi: 10.1007/s12272-013-0048-z.

33. Ma J, Hwang YK, Cho WH, Han SH, Hwang JK, Han JS. Macelignan attenuates activations of mitogen-activated protein kinases and nuclear factor kappa B induced by lipopolysaccharide in microglial cells. Biol Pharm Bull 2009; 32: 1085-1090, doi: 10.1248/bpb.32.1085.

34. Lee KE, Mun S, Pyun HB, Kim MS, Hwang JK. Effects of macelignan isolated from Myristica fragrans (Nutmeg) on expression of matrix metalloproteinase-1 and type I procollagen in UVB-irradiated human skin fibroblasts. Biol Pharm Bull 2012; 35: 1669-1675, doi: 10.1248/bpb.b12-00037.

35. Junttila MR, Li SP, Westermarck J. Phosphatase-mediated crosstalk between MAPK signaling pathways in the regulation of cell survival. FASEB $J$ 2008; 22: 954-965, doi: 10.1096/fj.06-7859rev.

36. Kraus PR, Fox DS, Cox GM, Heitman J. The Cryptococcus neoformans MAP kinase Mpk1 regulates cell integrity in response to antifungal drugs and loss of calcineurin function. Mol Microbiol 2003; 48: 1377-1387, doi: 10.1046/ j.1365-2958.2003.03508.x.

37. Kojima K, Bahn YS, Heitman J. Calcineurin, Mpk1 and Hog1 MAPK pathways independently control fludioxonil antifungal sensitivity in Cryptococcus neoformans. Microbiology 2006; 152: 591-604, doi: 10.1099/mic. $0.28571-0$

38. Jung KW, Bahn YS. The stress-activated signaling (SAS) pathways of a human fungal pathogen, Cryptococcus neoformans. Mycobiology 2009; 37: 161-170, doi: 10.4489/ MYCO.2009.37.3.161.

39. Babar SME, Islam MF, Rahman KMT, Sumi SS. Systems biology study of yeast mitogen activated protein kinase (MAPK) cascade for novel drug target identification against fungal pathogens. Int $\mathrm{J}$ Biosci Biochem Bioinformatics 2013; 3: 149-153, doi: 10.7763/IJBBB.2013.V3. 184.

40. Yogiara, Hwang SJ, Park S, Hwang JK, Pan JG. Food-grade antimicrobials potentiate the antibacterial activity of 1,2hexanediol. Lett Appl Microbiol 2015; 60: 431-439, doi: 10.1111/lam.12398. 